\title{
Gait Based Person Recognition Using Partial Least Squares Selection Scheme
}

\author{
Hema Mamidipaka $^{1}$ and Jagadeesh Gunti $^{2}$ \\ ${ }^{1}$ Department of ECE, JNTUK-UCEV, Vizianagaram, Andhra Pradesh, India \\ ${ }^{2}$ Department of ECE, JNTUK-UCEV, Vizianagaram, Andhra Pradesh, India
}

\begin{abstract}
The variations of viewing angle and intra-class of human beings have great impact on gait recognition systems. This work represents an Arbitrary View Transformation Model (AVTM) for recognizing the gait. Gait energy image (GEI) based gait authentication is effective approach to address the above problem, the method establishes an AVTM based on principle component analysis (PCA). Feature selection (FS) is performed using Partial least squares (PLS) method. The comparison of the AVTM PLS method with the existing methods shows significant advantages in terms of observing angle variation, carrying and attire changes. Experiments evaluated over CASIA gait database, shows that the proposed method improves the accuracy of recognition compared to the other existing methods.
\end{abstract}

\section{KEYWORDS}

Gait Analysis, GEI, PCA, PLS, Feature Selection, AVTM.

\section{INTRODUCTION}

Human gait is the most important biometric trait for person authentication. The biometric system mainly used to prevent the unauthorized access. Biometric resources such as face recognition, voice recognition, iris, fingerprints, palm prints, shoe prints and hand writing, are a subject of extensive research work, studied and employed in many applications. The advantage of gait as the biometric is that the gait of a human can be captured even from a great distance [1].

There is a need for automation in applications such as security systems, crime investigation department and surveillance. Today, biometric is an effective tool for reliable person authentication. The motion vision's main purpose is to use surveillance when unexpected occurrences befall us.

The classification of gait recognition is done in various ways. Human motion and vision is one of a kind, recognition based on a wearable sensor, through sensor information from floor of the motion are the other types. Wearable sensor systems require carrying the sensors and floor sensors system around that necessitates setting the sensors on the floor [2, 3]. First kind is further divided, based on appearance and model parameters and appearance method is divided into two categories: they are spatio-temporal and state-space methods. Most researchers used appearancebased method compared to model-based method [4]. 
International Journal on Cybernetics \& Informatics (IJCI) Vol. 5, No. 4, August 2016

The gait recognition system is capable of identifying humans from a distance beyond human interactions. This characteristic of gait recognition system is suitable for applications in large and controlled environments such as banks, military installations and even airports that are enabled to quickly detect threats.

\section{Problem Statement And Concepts}

\subsection{OBJECTIVE}

The primary objective of this paper is to evaluate the performance of different gait recognition methods selected for person identification.

\subsection{OPTIMUM GAIT ANALYSIS}

This section uses a PLS type of feature selection to find optimum gait analysis. In general human gait occurs in a periodic manner. Detection period helps in preserving the temporal information which reduces computational complexity and cost. Using the methods illustrated in [7,9] estimate the bounding box changes and the aspect ratio, which depends on the periodic changes in human walk. These methods use GEI as the important gait parameter. PLS method is applied to extract components of gait feature descriptor.

By the periodic estimation of the GEI features gives the gait information in temporal and spatial domain. Silhouettes are obtained through background subtraction and the GEI is obtained. $B_{l, t}(p, q)$ is a binary silhouette in which the pixels are located at position $(\mathrm{p}, \mathrm{q})$.Each binary silhouette has $l(l=1,2, \ldots \ldots \ldots L)$ gait cycles. Each gait cycle has $\mathrm{t}(\mathrm{t}=1,2 \ldots \mathrm{T})$ frames. Silhouette normalization is performed along both vertical and horizontal directions for a fixed range. GEI of height $\mathrm{M}$ and width $\mathrm{N}$ represents is given by

$$
f(p, q)=1 / T \sum_{l=1}^{L} \sum_{t=1}^{T} B_{l, t}(p, q)
$$

Here $\mathrm{T}$ represents total frames in one gait cycle. B represents the silhouette at $\mathrm{t}, \mathrm{p}$ and $\mathrm{q}$ are the coordinates of the image.

The 1-D feature vector is obtained by concatenating the value of each position in $B_{l, t}(p, q)$ along all consecutive rows and columns which is represented by $f_{k}^{m}$, where $\mathrm{k}$ represents the $k^{\text {th }}$ observing angle and $\mathrm{m}$ represents the $m^{\text {th }}$ subject. PLS regression is used as the FS scheme to learn about optimal feature representation and also used to reduce the dimension. The major advantage is reduction in dimension of target will not limit the class number considered in database of training set. Furthermore, on applying PLS and factorization process [7], it is found that optimized GEI is better than original GEI.

Consider the case of two different persons $\mathrm{m}$ and $\mathrm{n}$ with the same $k^{\text {th }}$ observing angle . Then $f_{k}^{m}$ and $f_{k}^{n}$ are the two sets of gait feature vectors are obtained. The objective function of maximum covariance between two variables is obtained by using PLS, which gives the optimal projection. The objective function is given by

$$
\max _{w_{k}}\left[\operatorname{COV}\left(f_{k}^{m} w_{k}, f_{k}^{n}\right)\right]
$$

For $k^{\text {th }}$ observing angle $w_{k}$ is the learned matrix of projection. The covariance of original GEI features with different observing angles is calculated using covariance operation. Therefore, from 
a given GEI feature vector $f_{k}^{m}$ we get the optimal feature vector $A_{k}^{m}$ for corresponding the $k^{\text {th }}$ angle [11].

$A_{k}^{m}=f_{k}^{m} w_{k}$

\subsection{AVTM PLS TECHNIQUE}

The following representation is regarding procedural steps for the AVTM PLS method for Gait authentication. Figure1. represents the block diagram of AVTM PLS method.

Step1 consider the gait sequence from the CASIA database.

Step2 Extract the frames from the Gait video sequence.

Step3 Perform the Background subtraction to extract the silhouette images [10].

Step4 Feature extraction is performed on silhouette images and gait features are found.

Step5 Estimate the gait period, by using this GEI is computed which is averaging of silhouettes over one gait period.

Step6 Dimensionality Reduction Techniques are used to reduce the higher dimensional feature to a lower dimensional feature. Which results in reduction in time, space and cost.

Step7 The reduced lower dimensional feature vectors are extracted by using singular value decomposition and then apply view transformation model with PLS.

Step8 The Extracted feature vectors are used for matching purpose. L1 norm distance is computed to find similarity of considered simplified gait.

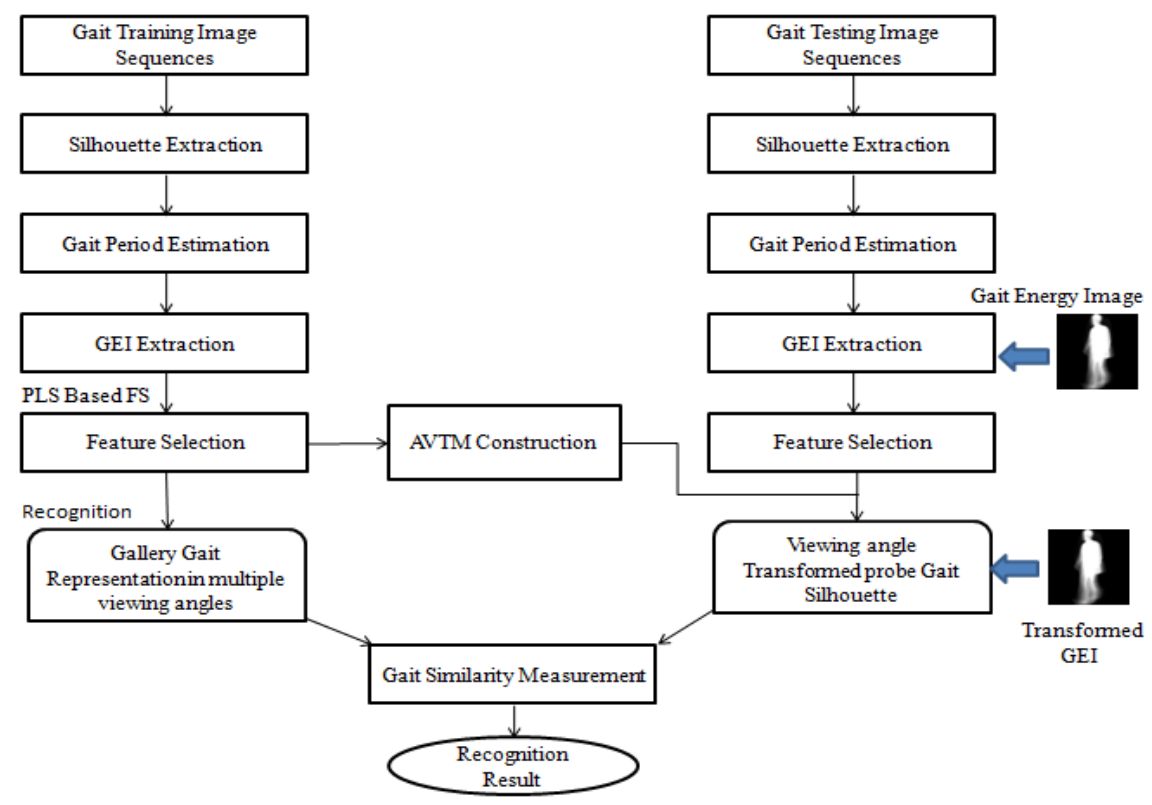

Figure1. Block diagram of Gait Recognition. 


\section{Modelling Of The Problem}

This section deals with modelling of Gait Recognition Method. It shows the analysis of the proposed method.

\subsection{Arbitrary View Transfromation Model}

The left hand side matrix of equation (4) is called optimized gait representation matrix represented as $A_{K}^{M}$. Every row indicates the gait data under same observing angle from different subjects whereas column represents same subject with different observing angles. Consider a total of $\mathrm{M}$ subjects and $\mathrm{K}$ observing angles and constructs a View Transformation Model. Singular Value Decomposition is used for factorization [7] as defined below:

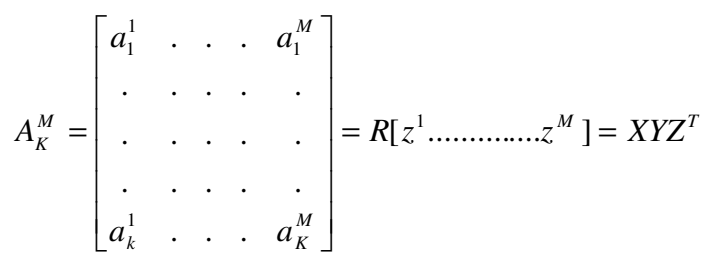

Here $\mathrm{X}$ and $\mathrm{Z}$ are orthogonal matrices with dimensions of $K N_{f} \times M, M \times M$ respectively. $a_{k}^{m}$ has the dimension $N_{f} \times 1$. S is a diagonal matrix with dimension $M \times M$ has the singular values. $R=\left[R_{1}, \ldots \ldots \ldots \ldots, R_{K}\right]^{T}=\mathrm{XY}$, here $R_{K}$ is sub-matrix of $\mathrm{XY}$ and $z^{m}$ is column vector.

The vector $z^{m}$ represents the gait feature vector of $m^{\text {th }}$ subject for any observing angle. Under a specific observing angle $R_{k}$ represents transforming matrix which is independent of subject. It helps in projecting shared gait feature vector $\mathrm{z}$ to gait feature vector under specific angle k. From equation (4), for an optimized gait vector $a_{j}^{m}$ from the $m^{\text {th }}$ subject with $j^{\text {th }}$ observing angle, the learned gait transformation of feature vectors from $j^{\text {th }}$ to $i^{\text {th }}$ observing angle is obtained as

$$
t_{a_{i}^{m}}=R_{i} R_{j}^{+} a_{j}^{m}
$$

Here, $R_{j}^{+}$represents pseudo inverse matrix.

\section{RESULTS}

\subsection{ObJective Measures And Simulation Platform}

The AVTM PLS method is compared with various View Transformation Models for same circumstance. Gait Energy Image features are extracted. Different methods have the variations among the VTM and FS algorithm. Consider the probe feature vector for various observing angles, the methods of AVTM and FS scheme are applied to obtain the shared subspace. Matching is performed using L1-norm distance.

Performance analysis is made by comparing recognition rate of different VTMs and its associate FS algorithm. Recognition rate gives the correctness in matching procedure. 
International Journal on Cybernetics \& Informatics (IJCI) Vol. 5, No. 4, August 2016

\subsection{GAIT SIMILARITY IN GAIT RECOGNITION}

A PLS based VTM along with factorization process is presented. The gait similarity measurement is simplified using L1- norm distance and is given by equation (8)

$$
d\left(a_{k}^{i}, a_{k}^{j}\right)=\left\|a_{k}^{i}-a_{k}^{j}\right\|
$$

Here $d$ represents the distance of separation between gait signatures of the two different persons under the same observing angle. The gait feature dimension is represented by $\mathrm{N}$. If the distance of separation is more then the similarity between the gait signatures $a_{k}^{i}$ and $a_{k}^{j}$.

\subsection{EXPERIMENTS}

Simulations are performed on CASIA gait database [3]. The dataset contains the data is obtained for all 11 degree observation angles from 124 subjects. From each observation angle six sequences are considered with normal walk [12]. Furthermore, this dataset comprises of two sequences indicating respective overcoat attire and bag carrying situation for all 11 observing angles.

\subsection{IDENTIFICATION RATE}

For a range of varying observing angle from $0^{\circ}$ to $180^{\circ}$ recognition rates of popular gait recognition methods are compared for various probe angles. From the figures 1, 2 and 3 represents the simulation results of the person with overcoat, with a bag and normal walk respectively.
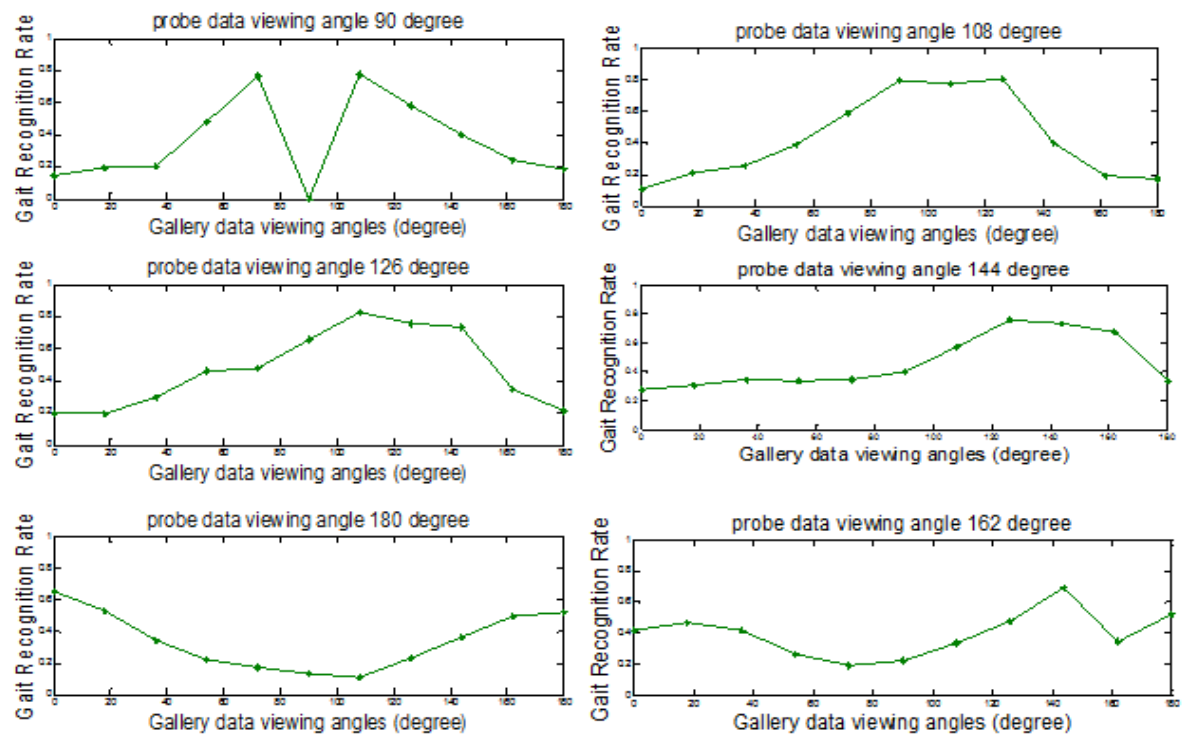

Fig:2

Figure2. Simulation results of the person with overcoat. 

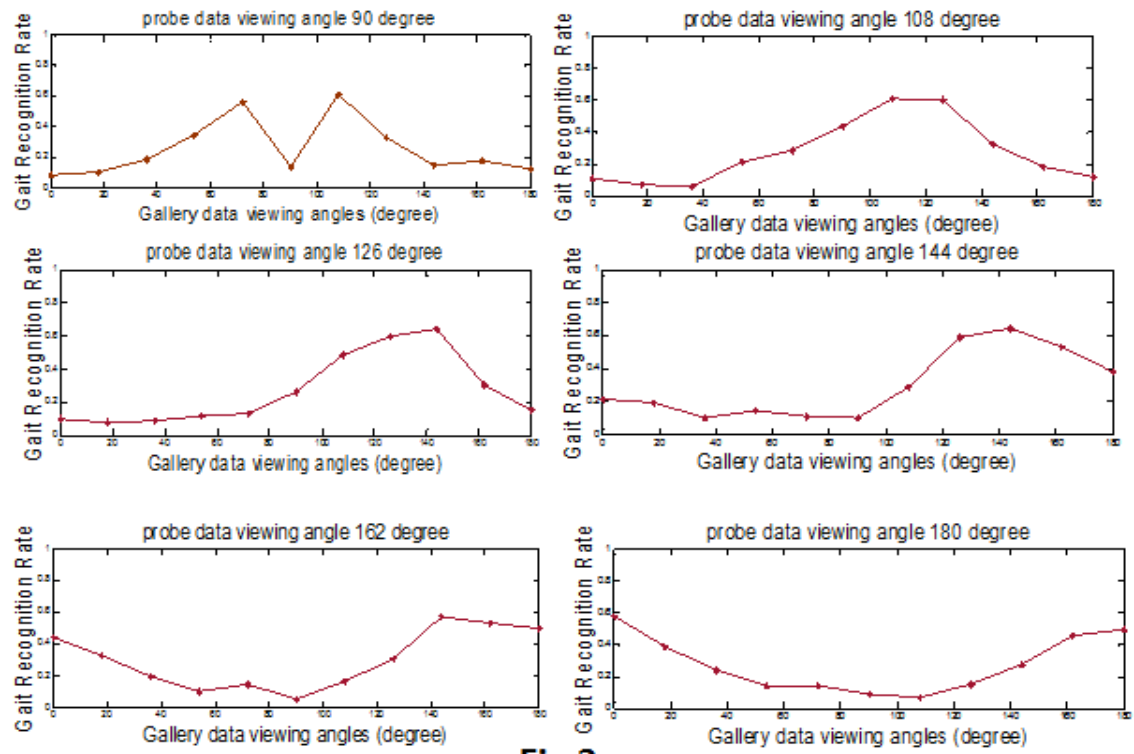

Fig:3

Figure 3. Simulation results of the person walk with a bag.
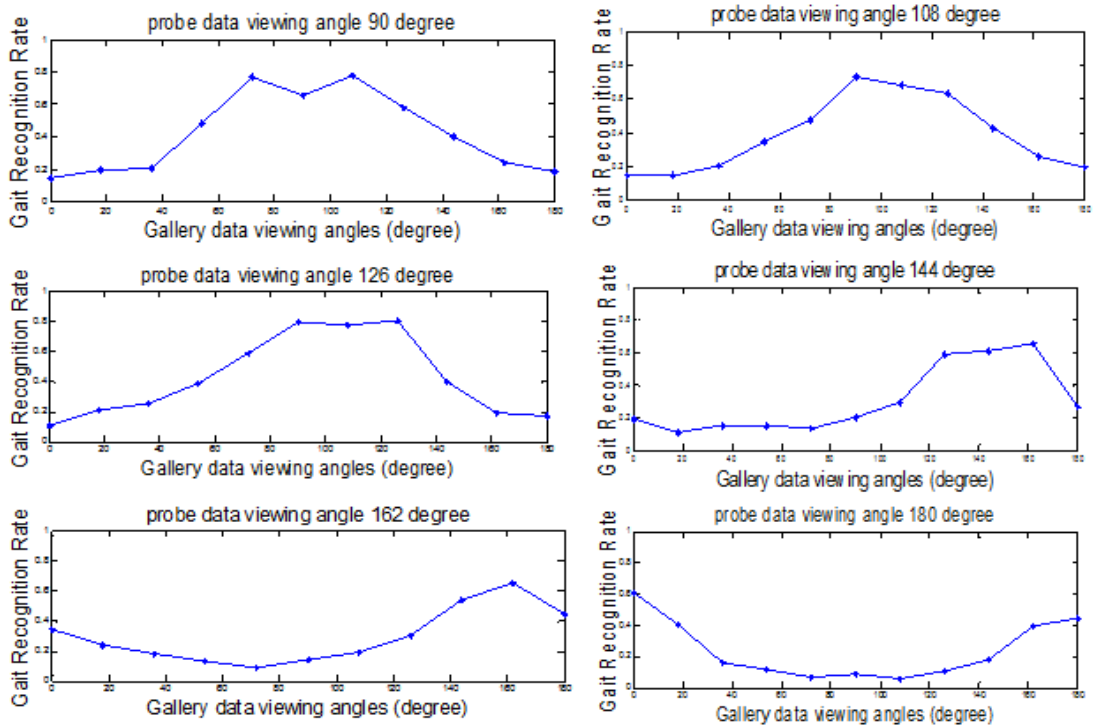

Fig:4

Figure 4. Simulation results of the person with normal walk.

From the Table number 1, 2, 3 and 4, the proposed technique is dominant in performance over [6], [7], [8] methods. Table1 represents the recognition rate of various algorithms for a given condition of a gallery data with observation angles varying from $72^{\circ}$ to $162^{\circ}$ where the probe gait data observation angle is given as $126^{\circ}$ (With overcoat).Table2 represents the recognition rate of various algorithms for a given condition of a gallery data with observation angles varying from $54^{\circ}$ to $144^{\circ}$ where the probe gait data observation angle is given as $90^{\circ}$ (With a bag). Table 3 represents the recognition rate of various algorithms for a given condition of a gallery data with 
International Journal on Cybernetics \& Informatics (IJCI) Vol. 5, No. 4, August 2016

observation angles varying from $54^{\circ}$ to $144^{\circ}$ where the probe gait data observation angle is given as $90^{\circ}$. Table 4 represents the recognition rate of various algorithms for a given condition of a gallery data with observation angles varying from $54^{\circ}$ to $144^{\circ}$ where the probe gait data observation angle is given as $144^{\circ}$.

Table 1. Various gait recognition algorithms and its accuracy of Recognition.

\begin{tabular}{|c|c|c|c|c|c|}
\hline Gallery observing angle & $72^{\circ}$ & $90^{\circ}$ & $108^{\circ}$ & $144^{\circ}$ & $162^{\circ}$ \\
\hline GEI+LDA+TSVD[7] & 0.09 & 0.10 & 0.20 & 0.30 & 0.13 \\
\hline Yu's method[8] & 0.14 & 0.09 & 0.06 & 0.18 & 0.02 \\
\hline AVTM_PLS & $\mathbf{0 . 4 8}$ & $\mathbf{0 . 6 5}$ & $\mathbf{0 . 8 5}$ & $\mathbf{0 . 7 8}$ & $\mathbf{0 . 2 8}$ \\
\hline
\end{tabular}

Table 2. Various gait recognition algorithms and its accuracy of Recognition.

\begin{tabular}{|c|c|c|c|c|c|}
\hline Gallery observing angle & $54^{\circ}$ & $72^{\circ}$ & $108^{\circ}$ & $126^{\circ}$ & $144^{\circ}$ \\
\hline GEI+LDA+TSVD[7] & 0.10 & 0.31 & 0.23 & 0.13 & 0.10 \\
\hline Yu's method[8] & 0.13 & 0.31 & 0.44 & 0.15 & 0.02 \\
\hline AVTM_PLS & $\mathbf{0 . 3 5}$ & $\mathbf{0 . 6 0}$ & $\mathbf{0 . 6 5}$ & $\mathbf{0 . 4 2}$ & $\mathbf{0 . 1 8}$ \\
\hline
\end{tabular}

Table 3. Various gait recognition algorithms and its accuracy of Recognition.

\begin{tabular}{|c|c|c|c|c|c|}
\hline Gallery observing angle & $54^{\circ}$ & $72^{\circ}$ & $108^{\circ}$ & $126^{\circ}$ & $144^{\circ}$ \\
\hline FG+SVD[6] & 0.29 & 0.40 & 0.45 & 0.30 & 0.20 \\
\hline GEI+LDA+TSVD[7] & 0.50 & 0.70 & 0.72 & 0.40 & 0.20 \\
\hline Rectified method[5] & $\mathbf{0 . 7 2}$ & 0.70 & 0.68 & 0.66 & - \\
\hline Yu's method[8] & 0.16 & 0.81 & 0.77 & 0.22 & 0.03 \\
\hline AVTM_PLS & 0.43 & $\mathbf{0 . 8 2}$ & $\mathbf{0 . 8 5}$ & $\mathbf{0 . 6 8}$ & $\mathbf{0 . 4 2}$ \\
\hline
\end{tabular}

Table 4. Various gait recognition algorithms and its accuracy of Recognition.

\begin{tabular}{|c|c|c|c|c|c|}
\hline Gallery observing angle & $54^{\circ}$ & $72^{\circ}$ & $90^{\circ}$ & $108^{\circ}$ & $144^{\circ}$ \\
\hline FG+SVD[6] & 0.20 & 0.29 & 0.48 & 0.60 & 0.40 \\
\hline GEI+LDA+TSVD[7] & 0.30 & 0.43 & 0.72 & 0.72 & 0.40 \\
\hline Rectified method[5] & $\mathbf{0 . 7 1}$ & 0.59 & 0.60 & 0.70 & - \\
\hline Yu's method[8] & 0.21 & 0.60 & 0.81 & 0.48 & 0.03 \\
\hline AVTM_PLS & 0.42 & $\mathbf{0 . 6 4}$ & $\mathbf{0 . 8 3}$ & $\mathbf{0 . 8 0}$ & $\mathbf{0 . 4 5}$ \\
\hline
\end{tabular}

\section{Conclusions}

All the VTMs using factorization are compared with proposed method. The accuracy of AVTM can be proved by considering the (GEI+TSVD) approach. The variation among Gait features with frequency- domain representation (FG) is used rather than GEI in [6]. PLS based AVTM feature selection algorithm is implemented for several view gait identification under various object positional angles like position of the bag and new objects like wearing of coat. For different observing angles the shared gait features are assumed with low significant performance on these multiple view gait databases with variations in carrying or wearing conditions. 
International Journal on Cybernetics \& Informatics (IJCI) Vol. 5, No. 4, August 2016

\section{REFERENCES}

[1] Gafurov, D., Snekkene, E. and Bours, P. 2007. Gait Authentication and Identification Using Wearable Accelerometer sensors. IEEE Workshop on automatic Identification Advanced Technologies, pp: 220-225

[2] Boulgouris, N.V., Hatzinakos, D. and Plataniotis, K. N. 2005. Gait Recognition: A Challenging Signal Processing Technology for Biometric Identification. Signal Processing Magazine, IEEE, pp: 78-90.

[3] Moeslunda, T. B., Adrian, H. and Volker, K. 2006. A survey of advances in vision based human motion capture and analysis, Computer Vision and Image Understanding, Volume 104, Issues 2- 3, November-December 2006, pp: 90-126.

[4] Bo, Y. and Yumei, W. 2006. A New Gait Recognition Method Based on Body Contour. Control, Automation Robotics and Vision, ICARCV International conference on, pp:1-6

[5] M.Goffredo, I.Bouchrika, J.N.Carter, and M.S.Nixon, "Self calibrating view-invariant gait bio metrics," IEEE Trans. On SMC- part B, 2010.

[6] J.Makihara, R.Sagawa, and Y.Mukaigawa, "Gait recognition using a view transformation model in the frequency domain," in ECCV, 2006.

[7] W.Kusakunniran, Q.Wu, H.Li, and J.Zhang, "Multiple view gait recognition using view transformation model based on optimized gait energy images," in ICCV workshops, 2009.

[8] S.Yu, D.Tan, and T.Tan, "A framework for evaluating the effect of view angle, clothing and carrying condition on gait recognition," in ICPR, 2006.

[9] L.Wang, T.Tan, H.Ning, and W.Hu, "Silhouette analysis based gait recognition for human identification," IEEE Trans. On PAMI, 2003.

[10] M. A. Hossain, Y. Makihara, J. Wang, and Y. Yagi, "Clothing-invariant gait identification using partbased clothing categorization and adaptive weight control," Pattern Recognit., vol. 43, no. 6, pp. 2281-2291, Jun. 2010.

[11] Shuai Zheng, Junge Zhang, Kaiqi Huang, Ran He, Tieniu Tan "Robust View Transformation Model for gait recognition".

[12] Zeng, Wei, Cong Wang and Feifei Yang. "Silhouette based gait recognition via deterministic learning", Pattern recognition, 2014.

[13] Kusakunniran, Worapan, Qiang Wu, Jian Zhang, Yi Ma and Hongdong Li. “ A New View-Invariant Feature For Cross View Gait Recognition", IEEE Transactions On Information Forensics and Security,2013.

[14] Wu, Zifeng, Yongzhen Huang and Liang wang . "Learning Representative Deep Features for image set analysis", IEEE Transactions on Multimedia, 2015.

[15] Pandey Neel Waleed Abdulla and Zoran Salsic. "Cepstral based featurs for gait recognition", 2102 11th International Conference on Information Science and Signal Processing and their Applications (ISSPA), 2012.

\section{AUTHORS}

M. Hema received M.Tech (DSCE) from JNTUA. She is currently pursuing Ph.D from JNTUK, Kakinada under the esteemed guidance of Prof. K. Babulu \& Prof. N. Balaji.

G. Jagadeesh received B. Tech degree from JNTUK. Currently pursuing M.Tech degree in UCEV, JNT University Kakinada.
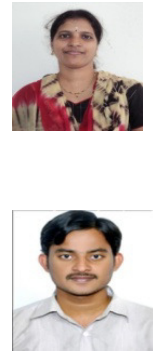